\title{
Uterus Size May Affect Reproductive Outcome in Women with Adenomyosis After Gnrh- Agonist Pretreatment Undergoing Frozen Embryo Transfer Cycles: A Retrospective Cohort Study
}

\section{Wei Xiong}

Peking Union Medical College Hospital

\section{Ruiyi Tang}

Peking Union Medical College Hospital

\section{Peng Wu}

Chinese Academy of Medical Sciences and Peking Union Medical College

\section{Zhengyi Sun}

Peking Union Medical College Hospital

jingran zhen ( $\sim$ zhenjingran002@sina.com)

Peking Union Medical College Hospital

Qi Yu

Peking Union Medical College Hospital

\section{Research}

Keywords: Gonadotropin-releasing hormone agonist, uterine enlargement, adenomyosis, live birth rate

Posted Date: December 10th, 2020

DOI: https://doi.org/10.21203/rs.3.rs-122461/v1

License: (c) (i) This work is licensed under a Creative Commons Attribution 4.0 International License.

Read Full License 


\section{Abstract}

Background: $\mathrm{GnRH}$-agonist is used to treat adenomyosis, but its efficacy in adenomyosis patients with uterine enlargement undergoing frozen embryo transfer (FET) is unclear.

Methods】The retrospective cohort study comprised 112 adenomyosis patients with uterine enlargement undergoing the first FET circle. A long-term GnRH-a pretreatment was administered to 112 patients with uterine enlargement. These patients were divided into two groups according to the therapeutic effect: patients with a normal-size uterus after $\mathrm{GnRH}$-a treatment (GN group) and patients with an enlarged uterus after $\mathrm{GnRH}$-a treatment (GL group).

Results囚Not all patients can shrink their uterus to a satisfactory level. After receiving $\mathrm{GnRH}-\mathrm{a}$ pretreatment, the uterus returned to normal size in $77 \%$ of patients (GN group), and $23 \%$ of patients had a persistently enlarged uterus (GL group). The pregnancy rate, clinical pregnancy rate, ongoing pregnancy rate, and live birth rate were significantly higher in the GN group than in the GL group. Controlling for the confounding factors, normal uterus size (odds ratio [OR] 4.50; $P=0.03$ ) and low body mass index (OR 3.13; $P=0.03$ ) affected the odds of achieving live birth. The cut-off value selected on the ROC curve of uterus volume after $\mathrm{GnRH}$-a treatment for detecting live birth was 144.7

Conclusions $\llbracket \mathrm{GnRH}$-a pretreatment was associated with the regression of adenomyosis lesions and improved clinical pregnancy outcomes in the adenomyosis patients with uterine enlargement whose lesion are $\mathrm{GnRH}$-a susceptible on FET cycles. However, about a quarter of patients may not be less responsive to $\mathrm{GnRH}-\mathrm{a}$ and have poorer pregnancy outcomes, especially in overweight women.

\section{Highlights}

1. The response to $\mathrm{GnRH}-\mathrm{a}$ was different in enlarged adenomyosis patients.

2. GnRH-a improved clinical pregnancy outcomes in the adenomyosis patients whose lesion are $\mathrm{GnRH}$ a susceptible.

3. About a quarter of patients may not be less responsive to $\mathrm{GnRH}-\mathrm{a}$ and have poorer pregnancy outcomes.

4. Overweight patients with enlarged adenomyosis may have poorer pregnancy outcomes.

\section{Introduction}

Adenomyosis is a common gynecological disorder that is defined as endometrial cells migrating into the myometrium, along with varying degrees of muscular hypertropia [1]. It is characterized by abnormal uterine bleeding, dysmenorrhea, and an enlarged uterus. Recently, because more women are delaying childbearing until their late $30 \mathrm{~s}$ or $40 \mathrm{~s}$, adenomyosis is being diagnosed more frequently in infertile patients undergoing in vitro fertilization (IVF). Previous studies have demonstrated that adenomyosis has a significant negative effect on the chance of implantation and is associated with a higher miscarriage 
rate and a lower live birth rate [2-4]. Treatment with a gonadotropin-releasing hormone agonist ( $\mathrm{GnRH}-\mathrm{a})$ has been widely used for adenomyosis. However, to date, there is insufficient evidence to suggest that $\mathrm{GnRH}$-a treatment would improve the pregnancy outcomes of women with adenomyosis undergoing IVF [5].

Uterine size is an important parameter in the assessment of the severity of adenomyosis. Uterine enlargement, as a consequence of the destruction of the normal architecture of the myometrium, reflects more severe adenomyosis. A larger uterus size indicates deeper and more extensive myometrial invasion; however, uterine volume has not been reported in most previous studies. To the best of our knowledge, no previous study has investigated whether $\mathrm{GnRH}$-a treatment improves implantation and pregnancy rates in adenomyosis patients with uterine enlargement. Thus, the purpose of this study was to examine the differences in outcomes of IVF with frozen embryo transfer (FET) cycles between adenomyosis patients with normal-size uterus and those with enlarged uterus undergoing $\mathrm{GnRH}$-a pretreatment and to identify predictive factors for live birth.

\section{Materials And Methods}

\section{Study design and data collection}

All adenomyosis patients with uterine enlargement who had initiated IVF cycles in the IVF program of the reproductive medical center of Peking Union Medical College Hospital between May 2012 and August 2019 and who met the inclusion criteria were evaluated. The inclusion criteria were as follows: indication for IVF/intracytoplasmic sperm injection (ICSI), no uterine malformations, and no endometrial abnormalities as assessed by transvaginal sonography (TVS). The exclusion criteria were as follows: focal adenomyosis, fibroids, endometrial polyps, and a history of myomectomy and adenomyotomy. Women who did not produce embryos or with incomplete data were also excluded. Only the first FET cycle for each patient was included (Fig. 1).

\section{IVF and laboratory procedures}

All women undergoing IVF for the first time underwent a pelvic examination and baseline TVS before commencing the FET cycle as per the standard protocol of our unit. Uterine enlargement was defined as a uterus size larger than that at week 6 of pregnancy by pelvic examination.

Patients were stimulated either by a $\mathrm{GnRH}-\mathrm{a}$ or an antagonist protocol. After ovarian stimulation and oocyte retrieval, insemination of all oocytes was performed using either standard insemination or ICSI. Patients could undergo one or more IVF cycles and freeze their embryos at the blastocyst stage.

The detailed vitrification and thawing protocol was conducted according to a previously described protocol [6]. Briefly, embryo vitrification was performed using a Cyrotop carrier system, in conjunction with dimethylsulfoxide-ethylene glycol-sucrose as a cryoprotectant. For thawing, embryos were transferred into a dilution solution in a sequential manner (1-0.5-0 mol/L sucrose). 
Cleavage-stage embryos (day 3) were graded according to the Cummins criteria [7]. Optimal day 3 embryos were placed in an extended culture to the blastocyst stage. On day 5 or 6 , embryos were assessed by a standardized scoring system on the expansion of the blastocele cavity and the integrity of both the inner cell mass and trophectoderm cells $[8,9]$.

In all FET cycles, no more than two embryos were transferred. All embryos were thawed on the day of transfer, and post-thaw embryos with $\geq 50 \%$ of blastomeres intact were considered to be surviving.

\section{FET protocol}

Patients with an enlarged uterus were treated with a depot preparation of the long-term GnRH-a, intramuscular Leuproreline acetate (Diphereline; Ipsen, France) $3.75 \mathrm{mg}$ every 28 days before the initiation of the FET procedures. The dose of $\mathrm{GnRH}$-a was determined on an individual basis according to the experience of the treating physicians. Prior to FET, all patients underwent TVS to reevaluate the uterine volume. The $\mathrm{GnRH}$-a group was divided into two subgroups according to the therapeutic effect: GN group comprising patients with a normal-size uterus after GnRH-a and GL group comprising patients with an enlarged uterus after $\mathrm{GnRH}$-a treatment.

The hormone replacement therapy protocol was initiated 20 days after the last $\mathrm{GnRH}$-a treatment. Patients were treated with oral estradiol valerate (3 mg twice daily; Progynova; Schering, Germany) to attain an endometrial thickness of $8 \mathrm{~mm}$. At that time, progesterone injection (Progesterone injection, Xianju Pharmaceutical Co. Ltd, Zhejiang, China) was commenced with a step-up from $20 \mathrm{mg}$ per day for 2 days to $40 \mathrm{mg}$ per day for 2 days and finally $40 \mathrm{mg}$ twice a day for 2 days. FET was performed on day 6 of progesterone administration. Patients were then followed up with weekly ultrasound examination until transfer of care at 12 weeks of gestational age. Progesterone supplementation was continued until 10 weeks of gestation.

\section{TVS of the uterus for the detection of adenomyosis}

Sonographic criteria for the diagnosis of adenomyosis were as follows: (1) asymmetry of the anterior or posterior myometrial wall, (2) diffuse abnormal echotexture of the myometrium with indistinctly marginated areas, abnormal echotexture presence of heterogeneity, or increased or decreased areas of echogenicity, (3) subendometrial echogenic linear striations, (4) subendometrial echogenic nodules, (5) globular-appearing uterus, (6) hyper-echogenic islands, or (7) presence of myometrial cysts [10].

The volume was calculated by measuring the maximum length $(\mathrm{L})$ and anteroposterior $(\mathrm{AP})$ and transverse diameters of the uterine corpus $(T)$ and using the formula for the volume of a prolate ellipsoid: $\vee=0.52 \diamond(L \diamond A P \diamond T)[11]$

\section{Outcome variables}

Pregnancy was confirmed by serum beta-human chorionic gonadotrophin ( $\beta \mathrm{hCG}$ ) assessment 7 days after FET. Clinical pregnancy was defined as the presence of an intrauterine gestational sac on TVS at 68 weeks of gestation. Biochemical pregnancy was defined as a serum $\beta$ hCG level of $5 \mathrm{mlU} / \mathrm{mL}$ in the 
absence of a gestational sac on TVS. Ongoing clinical pregnancy was defined as a clinical pregnancy that continued for 12 weeks of gestation. Live birth was defined as the delivery of an infant at $\geq 24$ weeks' gestation. Preterm delivery was defined as birth at $<37$ weeks' gestation. The implantation rate was defined as the number of gestational sacs observed divided by the number of embryos transferred. Clinical miscarriage was defined as loss occurring after the presence of an intrauterine gestational sac. Early miscarriage refers to a miscarriage that occurs before 12 weeks of gestation, while late miscarriage refers to a miscarriage occurring between 12-28 weeks of gestation.

\section{Statistical analyses}

Descriptive statistics were used to describe and compare the demographic and clinical characteristics of adenomyosis patients with or without $\mathrm{GnRH}$-a treatment. Continuous data were compared by Student's ttest or Mann-Whitney $U$ test, depending on the normal distribution status, and chi-squared or Fischer's exact test was performed to compare categorical variables. The analyses were performed in two stages. Initially, the separate association of each factor with live birth was examined in a series of univariable analyses. Subsequently, the combined effect of the variables on live birth was assessed in a multivariable analysis. In the first model, we assessed the differential effects of uterus size after $\mathrm{GnRH}$-a treatment on the risk of live birth as well as age, body mass index (BMI), number of transferred embryos, and embryo quality. In the second model, we assessed the independent effects of uterus size, with additional adjustment for age, BMI, number of transferred embryos, and embryo quality using multivariate logistic regression. Receiver-operating characteristic (ROC) curve analyses were used for the sensitivity and specificity estimates of the predictive role of uterus volume in identifying the likelihood of live birth. Appropriate cutoffs aimed at maximizing both sensitivity and specificity were identified. A $P$ value of < 0.05 was considered statistically significant. Statistical analyses were performed using the Statistical Package for Social Sciences version 23.0 (SPSS Inc.).

\section{Results}

A total of 112 typical adenomyosis patients with uterine enlargement and no history of adenomyotomy, undergoing the first FET cycle, were included in the data analysis. All patients were diagnosed using both ultrasonographic and clinical criteria. No patients underwent fresh embryo transfer. After receiving longterm $\mathrm{GnRH}$-a pretreatment, the uterus returned to normal size in 86 (77\%) patients (GN group), and the uterus was still enlarged in 26 (23\%) patients (GL group) before FET cycles.

The demographic data are summarized in Table 1. BMI and uterine volume before FET were significantly different between the study groups. The GN and GL groups had a BMI of $22.1 \pm 3.0 \mathrm{~kg} / \mathrm{m}^{2}$ and $24.2 \pm$ $4.4 \mathrm{~kg} / \mathrm{m}^{2}$, respectively $(P=0.01)$. The uterine volume after $\mathrm{GnRH}$-a was greater in the $\mathrm{GL}$ group (30.9 \pm $10.8 \mathrm{~mL}$ vs. $12.3 \pm 4.1 \mathrm{~mL} ; P \otimes 0.001)$. Numbers of transferred embryos, mean endometrial thickness on the day of embryo transfer, and embryo quality were similar between the two groups (Table 1).

The treatment outcome parameters are displayed in Table 2. The pregnancy rate $(71 \%$ vs. $42.3 \%, P=$ $0.01)$, clinical pregnancy rate $(53.5 \%$ vs. $30.8 \%, P=0.04)$, ongoing pregnancy rate $(40.7 \%$ vs. $23.1 \%, P=$ 
$0.03)$, and live birth rate ( $39.5 \%$ vs. $11.5 \%, P=0.01)$ were significantly higher in the GN group than in the GL group. Moreover, four women in the GL group had miscarriage at 20-24 weeks gestation, while only one woman in the GN group experienced miscarriage during this period. Women in the GN group had a significantly lower miscarriage rate in the second trimester of pregnancy than those in the GL group (1.2\% vs. $11.5 \%, P=0.01)$. There were no statistically significant differences between the groups in biochemical pregnancy, ectopic pregnancy, early miscarriage, premature delivery, and implantation rate $(P>0.05)$ (Table 2).

The results of the univariate and multivariate analyses examining the associations between demographic and clinical variables and live birth are shown in Table 3. BMI, number of transferred embryos, embryo quality, and uterus size after $\mathrm{GnRH}$-a treatment were significantly associated with live birth in the univariate logistic regression models, whereas age and endometrium thickness were not associated with live birth. After multivariate logistic regression analysis, $\mathrm{BMI}<24$, embryo quality $\geq 4 \mathrm{BB}$, and a normal uterus size after $\mathrm{GnRH}$-a retained their significant effects on live birth after adjusting for the confounding factors. A normal uterus size had the greatest effect on the odds of achieving live birth (odds ratio 4.50; $95 \%$ confidence interval, $1.13-17.92 ; P=0.03$ )

ROC curves revealed that uterus size was predictive of the presence of live birth with a cut-off value of 144.7 (area under the curve $=0.68 ; 56.0 \%$ sensitivity and $73 \%$ specificity; $P=0.002$ ) (Fig. 2 ).

\section{Discussion}

Nowadays, adenomyosis is frequently encountered in women undergoing IVF [12]. The use of GnRH-a treatment for adenomyosis and its effect on fertility are debatable. In the present study, GnRH-a treatment had different effects on pregnancy outcomes because of different therapeutic responses in patients with enlarged adenomyosis. The group more responsive to GnRH-a (GN group) was shown to be associated with increased clinical pregnancy rate, ongoing pregnancy rate, and live birth rate and lower late miscarriage rate compared with the less responsive group (GL group). The implantation rate was also higher in the more responsive group (GN group) than in the less responsive group (GL group), although the difference did not reach statistical significance.

Some studies $[4,5]$ reported that pretreatment with $\mathrm{GnRH}$-a has no benefit in improving IVF outcomes in adenomyosis. Notably, these studies focused on fresh embryo transfer cycles. The hyperestrogenic state resulting from subsequent controlled ovarian stimulation (COS) may diminish the effect of $\mathrm{GnRH}$-a and even aggravate adenomyosis. Furthermore, women with adenomyosis are often in their later reproductive years and have inherently reduced ovarian reserves. Ovarian suppression with a multi-dose $\mathrm{GnRH}$-a may negate its effect on the ovarian response, which might be an additional cause of impaired treatment efficacy. Therefore, IVF with FET may be preferable in these patients. The association between pretreatment with a GnRH-a and pregnancy outcomes in FET cycles may not be affected by ovarian stimulation regimens. Moreover, treatment with $\mathrm{GnRH}$-a during FET cycles does not affect the dose of gonadotropin or the duration of COS. Recently, a few studies have demonstrated the positive effect of 
long-term $\mathrm{GnRH}$-a administration before FET on the pregnancy outcomes of infertile women with adenomyosis and a normal sized uterus. In a study by Niu et al. [13], clinical pregnancy, implantation, and ongoing pregnancy rates were significantly higher in women who were pretreated with $\mathrm{GnRH}$-a than in those who were not. Hebisha et al. [14] reported a significant increase in the implantation and pregnancy rates when $\mathrm{GnRH}$-a was administered prior to estrogen-progesterone preparation of the endometrium in FET compared to when it was not. These studies indicate some beneficial effects of GnRH-a therapy in women with adenomyosis, including a potential for improved implantation. Our study extends these findings to demonstrate that this effect of $\mathrm{GnRH}-\mathrm{a}$ administration is more pronounced in patients undergoing FET who achieve a normal-size uterus after $\mathrm{GnRH}$-a pretreatment than in those with an enlarged uterus even after $\mathrm{GnRH}$-a pretreatment.

The adenomyotic uterus provides a dysfunctional environment for the maintenance of pregnancy $[2,15]$. Mechanisms may include uterine dysperistalsis, abnormal concentrations of free radicals in the uterine environment, and altered endometrial vascularization. All the factors would otherwise be in direct contact with invading trophoblast cells [16-19]. Evidence has confirmed that $\mathrm{GnRH}$-a promotes a decrease in the size and demarcation of adenomyotic lesions in the uterus [20,21]. The pathomechanism of GnRH-a therapy on adenomyosis is multifactorial and is suggested to involve a hypo-estrogenic effect, which reduces angiogenesis, inhibits cell proliferation, induces apoptosis, and decreases vascular endothelial growth factor (VEGF) secretion in the myometrium [22-24]. Additionally, a previous study using a mouse model of adenomyosis revealed that $\mathrm{GnRH}$-a treatment might improve pregnancy outcomes by restoring endometrial receptivity [25]. Our results suggest that the reduction in uterine size in response to $\mathrm{GnRH}-\mathrm{a}$ administration increases the probability of live birth in adenomyosis patients undergoing IVF with FET. It is theoretically possible that the greater reduction in uterine size after $\mathrm{GnRH}$-a use may relate to increased medical responsiveness. Moreover, the ultra-long downregulation has been demonstrated to reduce early pregnancy loss in the setting of adenomyosis. We found that the uterine volume after GnRH-a treatment may account for late miscarriage owing to anatomical and hormonal factors.

It is worth noting that $23 \%$ of patients still had an enlarged uterus after adequate doses of $\mathrm{GnRH}-\mathrm{a}$, and the live birth rates of these patients were relatively low in our study. A possible explanation for this observation is that $\mathrm{GnRH}$-a has a weaker anti-proliferative effect on adenomyosis cells in these patients. Our results are supported by the study by Khan et al. [26], who found that $\mathrm{GnRH}$-a did not affect cell growth in $30 \%$ of women with adenomyosis and observed a lower cellular response to $\mathrm{GnRH}-\mathrm{a}$ in adenomyosis cells than in cells derived from endometriosis and fibroids. This suggests that other pretreatment options, such as surgery, should be considered when $\mathrm{GnRH}-\mathrm{a}$ administration is not satisfactory. The cut-off value selected on the ROC curve of uterus volume after $\mathrm{GnRH}$-a treatment for detecting live birth was 144.7.

Our study demonstrated that adenomyosis patients in the GN group had a significantly higher BMI than those in the GL group. Overweight patients with enlarged adenomyosis may have poorer pregnancy outcomes. While earlier studies have concluded that obesity is closely correlated with the development of adenomyosis [2], our finding extends these claims by indicating that high BMI may be a risk factor for 
less responsiveness to $\mathrm{GnRH}$-a treatment for enlarged adenomyosis. [27] This may be because estradiol levels arising from the peripheral adipose tissue negated the desired hypoestrogenic effect of $\mathrm{GnRH}-\mathrm{a}$ administration. These results are potentially useful for pre-IVF counseling of women with adenomyosis and uterine enlargement.

The main strength of this study is the inclusion of a study population that was strictly limited to enlarged adenomyosis patients. The globular enlargement of the uterus is the most common feature of adenomyosis, which can be easily identified clinically and sonographically. And primary outcome and only the results regarding the first cycle should be considered. Thus, strict selection criteria reduced the heterogeneity of the study population. Moreover, the study was restricted to FET, which is an important area that deserves more research. Finally, multivariable logistic regression models were used to control for potential confounders.

Nevertheless, this study also has some limitations. First, this was a retrospective study. Second, as this study included patients who wanted to become pregnant, histological confirmation of adenomyosis was not possible. Nonetheless, we do not think that this significantly affected our results as TVS is an accepted and accurate noninvasive method for diagnosing adenomyosis. TVS has been demonstrated to have a sufficiently high diagnostic accuracy in adenomyosis cases, with a reported sensitivity of $82.5 \%$ and a specificity of $84.6 \%$ [10]. Moreover, TVS is a cost-effective, less invasive, and readily available tool for obstetricians and gynecologists. Uterine size is routinely measured during ultrasound examination, and the ultrasonographic features of adenomyosis have been clearly defined. Third, there was no control group of patients with adenomyosis and an enlarged uterus who were not pretreated with GnRH-a. However, this would not have been ethical given the strong evidence favoring long-term GnRH-a use.

In conclusion, we found that GnRH-a administration before FET for adenomyosis could be an effective treatment option for adenomyosis patients with uterine enlargement whose lesions are $\mathrm{GnRH}-\mathrm{a}$ susceptible. However, approximately a quarter of adenomyosis patients with an enlarged uterus may be less responsive to $\mathrm{GnRH}-\mathrm{a}$, especially overweight women. When the reduction of uterine size after treatment is not obvious, other treatment methods should be considered. A subsequent prospective randomized controlled study is required to provide conclusive evidence of the benefit of this approach.

\section{Declarations}

\section{-Ethical Approval and Consent to participate:}

This study was conducted in accordance with the guidelines of the Declaration of Helsinki and approved by the Ethics Committee of Peking Union Medical College Hospital (S-K 1360). The requirement for written informed consent was waived due to the retrospective nature of this study.

\section{- Consent for publication:}




\section{- Availability of supporting data:}

The Ethical Review Board approval was obtained for public sharing and presentation of data on group level only. This means that the data used in this study can only be used for the approved research and cannot be shared by the authors.

\section{- Competing interests:}

The authors declare that they have no competing interests.

\section{- Funding:}

Not applicable

\section{- Authors' contributions:}

WX, JZ and QY conceived and designed the study. RT, PW and ZS were responsible for coordinating the study. WX collected the data, conducted the analysis, and drafted the manuscript. JZ supervised the study and reviewed the first draft of the manuscript. All authors critically revised the manuscript and approved the final manuscript.

\section{- Acknowledgements:}

We would like to acknowledge Dr. Penghui Feng for his guidance on the statistical analysis, and to thank Yanan Wang for their contributions to data collection regarding this study

\section{References}

1. Ferenczy, A., Pathophysiology of adenomyosis. Hum Reprod Update, 1998. 4(4): p. 312-22.

2. Stanekova, V., R.J. Woodman, and K. Tremellen, The rate of euploid miscarriage is increased in the setting of adenomyosis. Hum Reprod Open, 2018. 2018(3): p. hoy011.

3. Younes, G. and T. Tulandi, Effects of adenomyosis on in?vitro fertilization treatment outcomes: a meta-analysis. Fertility and Sterility, 2017. 108(3): p. 483-490.e3.

4. Paolo, V., et al., Uterine adenomyosis and in vitro fertilization outcome: a systematic review and meta-analysis. Human Reproduction, 2014(5): p. 964-977. 
5. Chen, M., et al., Impact of Gonadotropin-Releasing Hormone Agonist Pre-treatment on the Cumulative Live Birth Rate in Infertile Women With Adenomyosis Treated With IVF/ICSI: A Retrospective Cohort Study. Front Endocrinol (Lausanne), 2020. 11: p. 318.

6. Testart, J., et al., High pregnancy rate after early human embryo freezing. Fertil Steril, 1986. 46(2): p. 268-72.

7. Cummins, J.M., et al., A formula for scoring human embryo growth rates in in vitro fertilization: its value in predicting pregnancy and in comparison with visual estimates of embryo quality. J In Vitro Fert Embryo Transf, 1986. 3(5): p. 284-95.

8. The Istanbul consensus workshop on embryo assessment: proceedings of an expert meeting. Hum Reprod, 2011. 26(6): p. 1270-83.

9. Gardner, D.K., et al., Blastocyst score affects implantation and pregnancy outcome: towards a single blastocyst transfer. Fertil Steril, 2000. 73(6): p. 1155-8.

10. Meredith, S.M., L. Sanchez-Ramos, and A.M. Kaunitz, Diagnostic accuracy of transvaginal sonography for the diagnosis of adenomyosis: systematic review and metaanalysis. Am J Obstet Gynecol, 2009. 201(1): p. 107.e1-6.

11. Goldstein, S.R., et al., Estimation of nongravid uterine volume based on a nomogram of gravid uterine volume: its value in gynecologic uterine abnormalities. Obstet Gynecol, 1988. 72(1): p. 86-90.

12. Dueholm, M., Uterine adenomyosis and infertility, review of reproductive outcome after in vitro fertilization and surgery. Acta Obstet Gynecol Scand, 2017. 96(6): p. 715-726.

13. Niu, Z., et al., Long-term pituitary downregulation before frozen embryo transfer could improve pregnancy outcomes in women with adenomyosis. Gynecol Endocrinol, 2013. 29(12): p. 1026-30.

14. Hebisha, S.A. and H.M. Adel, GnRh Agonist Treatment Improves Implantation and Pregnancy Rates of Frozen-Thawed Embryos Transfer. J Obstet Gynaecol India, 2017. 67(2): p. 133-136.

15. Martínez-Conejero, J.A., et al., Adenomyosis does not affect implantation, but is associated with miscarriage in patients undergoing oocyte donation. Fertil Steril, 2011. 96(4): p. 943-50.

16. Kissler, S., et al., Uterotubal transport disorder in adenomyosis and endometriosis-a cause for infertility. Bjog, 2006. 113(8): p. 902-8.

17. Benson, R.C. and V.D. Sneeden, Adenomyosis: a reappraisal of symptomatology. Am J Obstet Gynecol, 1958. 76(5): p. 1044-57; discussion 1057-61.

18. Pontis, A., et al., Adenomyosis: a systematic review of medical treatment. Gynecol Endocrinol, 2016. 32(9): p. 696-700.

19. Kuijsters, N.P.M., et al., Uterine peristalsis and fertility: current knowledge and future perspectives: a review and meta-analysis. Reprod Biomed Online, 2017. 35(1): p. 50-71.

20. Imaoka, I., et al., MR imaging of diffuse adenomyosis changes after GnRH analog therapy. J Magn Reson Imaging, 2002. 15(3): p. 285-90.

21. Nelson, J.R. and S.L. Corson, Long-term management of adenomyosis with a gonadotropin-releasing hormone agonist: a case report. Fertil Steril, 1993. 59(2): p. 441-3. 
22. Khan, K.N., et al., Changes in tissue inflammation, angiogenesis and apoptosis in endometriosis, adenomyosis and uterine myoma after GnRH agonist therapy. Hum Reprod, 2010. 25(3): p. 642-53.

23. Huang, F., et al., Effect of GnRH-II on the ESC proliferation, apoptosis and VEGF secretion in patients with endometriosis in vitro. Int J Clin Exp Pathol, 2013. 6(11): p. 2487-96.

24. de Ziegler, D., et al., Optimal uterine anatomy and physiology necessary for normal implantation and placentation. Fertil Steril, 2016. 105(4): p. 844-54.

25. Guo, S., et al., GnRH agonist improves pregnancy outcome in mice with induced adenomyosis by restoring endometrial receptivity. Drug Des Devel Ther, 2018. 12: p. 1621-1631.

26. Khan, K.N., et al., Cell proliferation effect of GnRH agonist on pathological lesions of women with endometriosis, adenomyosis and uterine myoma. Hum Reprod, 2010. 25(11): p. 2878-90.

27. Trabert, B., et al., A case-control investigation of adenomyosis: impact of control group selection on risk factor strength. Womens Health Issues, 2011. 21(2): p. 160-4.

\section{Tables}


Table 1

Baseline characteristics of study participants.

\begin{tabular}{|c|c|c|c|}
\hline & \multicolumn{3}{|c|}{ Adenomyosis } \\
\hline & $\begin{array}{l}\text { GN } \\
\text { group } \\
(n=86)\end{array}$ & $\begin{array}{l}\text { GL } \\
\text { group } \\
(n=26)\end{array}$ & P-value \\
\hline Age (years) & $36.1 \pm 3.6$ & $36.1 \pm 4.6$ & 0.99 \\
\hline $\mathrm{BMI}\left(\mathrm{kg} / \mathrm{m}^{2}\right)$ & $22.1 \pm 3.0$ & $24.2 \pm 4.4$ & 0.01 \\
\hline Infertility classification & & & 0.24 \\
\hline No. of patients with primary infertility & 41 & 9 & \\
\hline No. of patients with secondary infertility & 45 & 17 & \\
\hline $\begin{array}{l}\text { Main indication of IVF/ICSI } \\
\text { Male factor, } \mathrm{n}(\%) \\
\text { Tubal factor, } \mathrm{n}(\%) \\
\text { Endometriosis factor, } \mathrm{n}(\%) \\
\text { Unexplained, } \mathrm{n}(\%)\end{array}$ & $\begin{array}{l}19(22 \%) \\
25(29 \%) \\
38(44 \%) \\
4(5 \%)\end{array}$ & $\begin{array}{l}5(19 \%) \\
7(27 \%) \\
13(50 \%) \\
1(4 \%)\end{array}$ & 0.96 \\
\hline Years of infertility & $5.4 \pm 2.7$ & $4.4 \pm 2.0$ & 0.09 \\
\hline Technique used & & & 0.56 \\
\hline IVF & 74 & 21 & \\
\hline ICSI & 12 & 5 & \\
\hline Uterine volume $(\mathrm{mL})$ before $\mathrm{GnRH}-\mathrm{a}$ & $56.7 \pm 32.3$ & $64.9 \pm 26.1$ & 0.88 \\
\hline Uterine volume $(\mathrm{mL})$ after $\mathrm{GnRH}-\mathrm{a}$ & $12.3 \pm 4.1$ & $30.9 \pm 10.8$ & 0.00 \\
\hline No. of transferred embryos & & & 0.11 \\
\hline $1, \mathrm{n}(\%)$ & $17(20 \%)$ & $9(35 \%)$ & \\
\hline $2, \mathrm{n}(\%)$ & $69(80 \%)$ & $17(65 \%)$ & \\
\hline $\begin{array}{l}\text { Embryos quality at blastocyst stage } \\
\geq 4 \mathrm{BB}, \mathrm{n}(\%) \\
<4 \mathrm{BB}, \mathrm{n}(\%)\end{array}$ & $\begin{array}{l}88(57 \%) \\
67(43 \%)\end{array}$ & $\begin{array}{l}22(51 \%) \\
21(49 \%)\end{array}$ & 0.51 \\
\hline Endometrium thickness at embryo transfer $(\mathrm{cm})$ & $11.4 \pm 2.4$ & $11.8 \pm 3.3$ & 0.44 \\
\hline
\end{tabular}


Table 2

Treatment outcomes of two groups of patients.

\begin{tabular}{|llll|}
\hline \multicolumn{3}{|c|}{ Adenomyosis } & P-value \\
\hline Pregnancy Outcomes & $\begin{array}{l}\text { GN group } \\
(\mathrm{n}=86)\end{array}$ & $\begin{array}{l}\text { GL group } \\
(\mathrm{n}=26)\end{array}$ & \\
\hline Pregnancy, $\mathrm{n}(\%)$ & $61(71 \%)$ & $11(42.3 \%)$ & 0.01 \\
\hline Clinical pregnancy, $\mathrm{n}(\%)$ & $46(53.5 \%)$ & $8(30.8 \%)$ & 0.04 \\
\hline Biochemical pregnancy, $\mathrm{n}(\%)$ & $14(16.3 \%)$ & $3(11.5 \%)$ & 0.56 \\
\hline Ongoing pregnancy, $\mathrm{n}(\%)$ & $35(40.7 \%)$ & $6(23.1 \%)$ & 0.03 \\
\hline Ectopic pregnancy, $\mathrm{n}(\%)$ & $1(1.1 \%)$ & $0(0 \%)$ & 0.58 \\
\hline Live birth, $\mathrm{n}(\%)$ & $34(39.5 \%)$ & $3(11.5 \%)$ & 0.01 \\
\hline Premature delivery, $\mathrm{n}(\%)$ & $4(4.7 \%)$ & $1(3.8 \%)$ & 0.86 \\
\hline Implantation rate, $\mathrm{n}(\%)$ & $58 / 155(37.4 \%)$ & $10 / 43(23.3 \%)$ & 0.08 \\
\hline Clinical miscarriage, $\mathrm{n}(\%)$ & $12(14.0 \%)$ & $5 / 26(19.2 \%)$ & 0.51 \\
\hline Early miscarriage, $\mathrm{n}(\%)$ & $11(12.8 \%)$ & $2(7.7 \%)$ & 0.48 \\
\hline Late miscarriage, $\mathrm{n}(\%)$ & $1(1.2 \%)$ & $3(11.5 \%)$ & 0.01 \\
\hline
\end{tabular}


Table 3

Univariate and multivariate analysis of demographic and clinical parameters for the prediction of Live birth in patients with enlarged adenomyosis.

\begin{tabular}{|c|c|c|c|c|c|}
\hline & & \multicolumn{2}{|c|}{ Univariate analysis } & \multicolumn{2}{|c|}{ Multivariate analysis } \\
\hline & $\begin{array}{l}\text { Live birth } \\
\mathrm{n}(\%)\end{array}$ & $\begin{array}{l}\mathrm{OR} \\
(95 \% \mathrm{Cl})\end{array}$ & $\begin{array}{l}P \text { - } \\
\text { value }\end{array}$ & $\begin{array}{l}\mathrm{OR} \\
(95 \% \mathrm{Cl})\end{array}$ & P-value \\
\hline Age & - & $\begin{array}{l}0.99(0.89- \\
1.10)\end{array}$ & 0.79 & $1.00(0.89-1.14)$ & 0.95 \\
\hline $\begin{array}{l}\mathrm{BMI} \\
\geq 24^{\star \star} \\
<24\end{array}$ & $\begin{array}{l}7 / 36(19 \%) \\
30 / 76(39 \%)\end{array}$ & $\begin{array}{l}\text { Reference } \\
2.70(1.05- \\
6.95)\end{array}$ & 0.04 & $\begin{array}{l}\text { Reference } \\
3.13(1.10-8.93)\end{array}$ & 0.03 \\
\hline $\begin{array}{l}\text { Endometrium } \\
\text { thickness }\end{array}$ & & $\begin{array}{l}1.07(0.92- \\
1.24)\end{array}$ & 0.37 & $1.07(0.90-1.28)$ & 0.43 \\
\hline $\begin{array}{l}\text { No. of transferred } \\
\text { embryos }\end{array}$ & & & 0.04 & & 0.40 \\
\hline 1 & $4 / 26(15 \%)$ & Reference & & Reference & \\
\hline 2 & $33 / 86(38 \%)$ & $\begin{array}{l}3.43(1.08- \\
10.8)\end{array}$ & & $1.76(0.47-6.63)$ & \\
\hline $\begin{array}{l}\text { Embryos quality } \geq \\
\text { 4BB }\end{array}$ & & & 0.01 & & 0.03 \\
\hline No & 6/36(17\%) & Reference & & Reference & \\
\hline Yes & $31 / 76(41 \%)$ & $\begin{array}{l}3.44(1.28- \\
9.26)\end{array}$ & & $3.59(1.13-11.37)$ & \\
\hline $\begin{array}{l}\text { Uterus size after } \\
\text { treatment } \\
\text { Enlarged* } \\
\text { Normal size }\end{array}$ & $\begin{array}{l}3 / 26(12 \%) \\
34 / 86(40 \%)\end{array}$ & $\begin{array}{l}\text { Reference } \\
5.01(1.40- \\
18.00)\end{array}$ & 0.01 & $\begin{array}{l}\text { Reference } \\
4.50(1.13-17.92)\end{array}$ & 0.03 \\
\hline \multicolumn{6}{|c|}{ GnRH-a, GnRH- agonist; FET, frozen embryo transfer; OR: Odd ratio; BMI, body mass index } \\
\hline \multicolumn{6}{|c|}{$\begin{array}{l}\text { * Uterine enlargement was defined as the size of the uterus over the sixth week of pregnancy by pelvic } \\
\text { examination. }\end{array}$} \\
\hline$\star *$ Using the Chines & iteria, overw & ght is defined & $\mathrm{MI} \geq 2$ & & \\
\hline
\end{tabular}

\section{Figures}




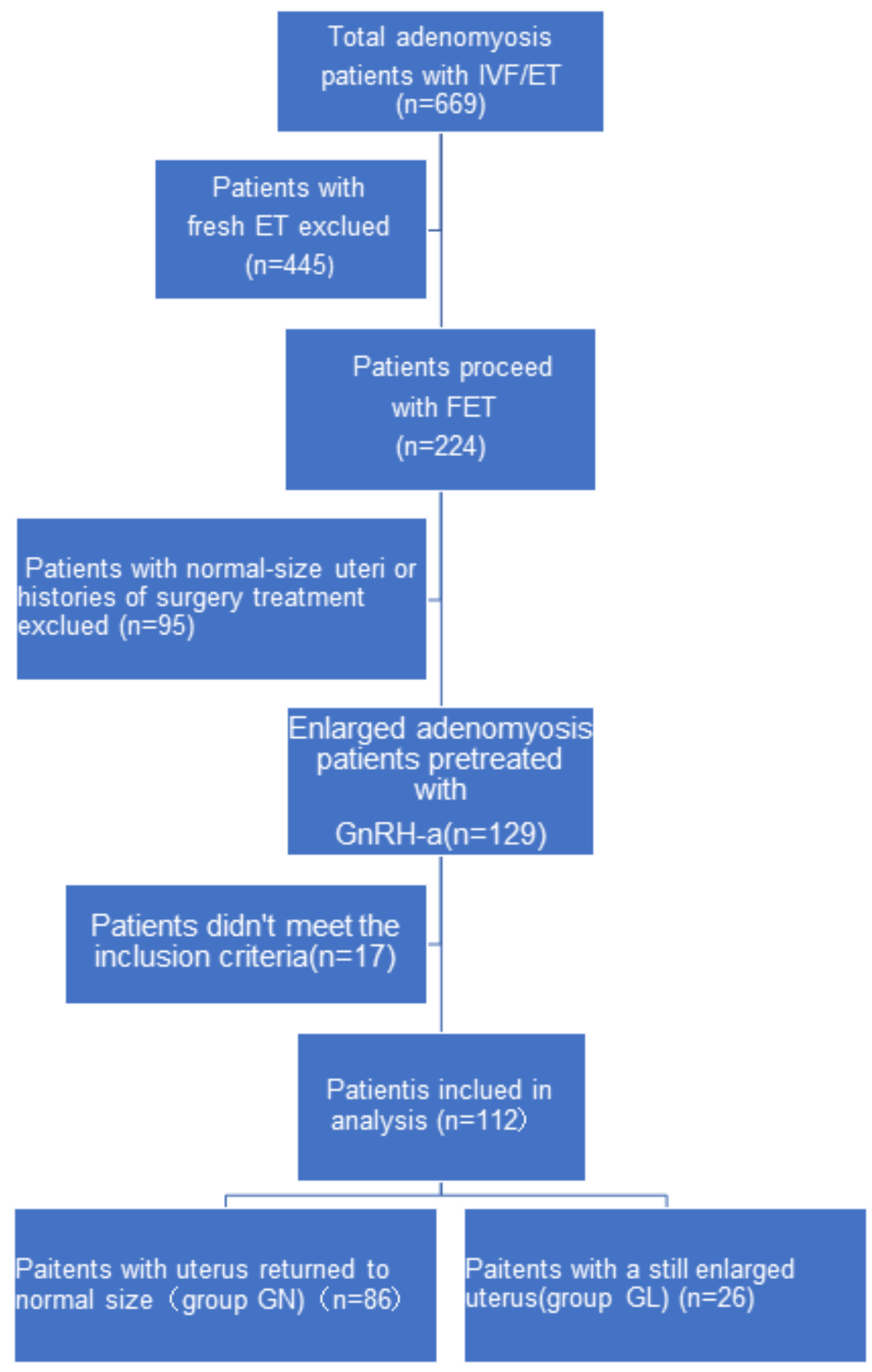

Figure 1

Study flow diagram. 


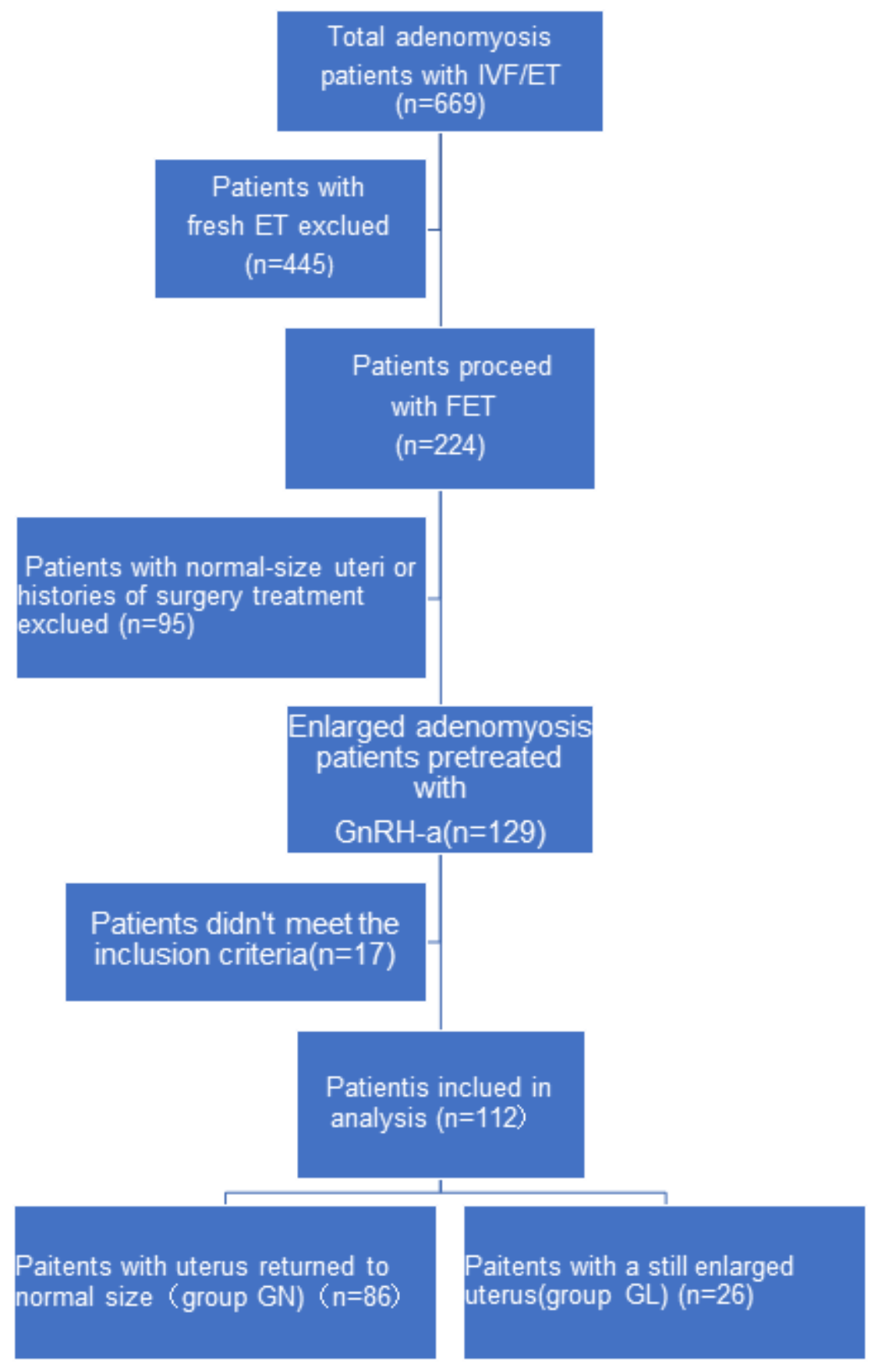

Figure 1

Study flow diagram. 


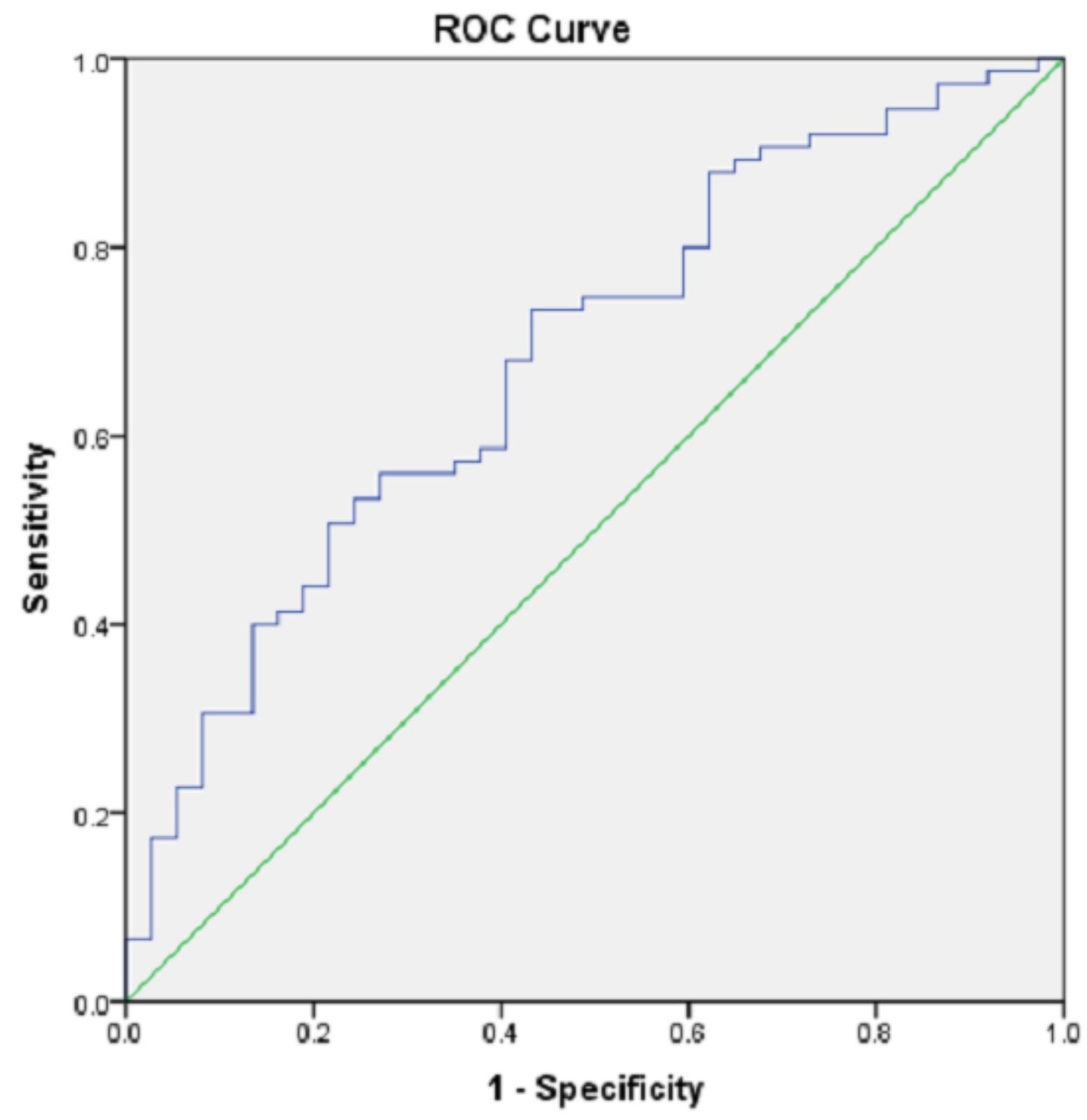

Figure 2

Receiver-operating characteristic curve of uterus volume against detection of live birth. 


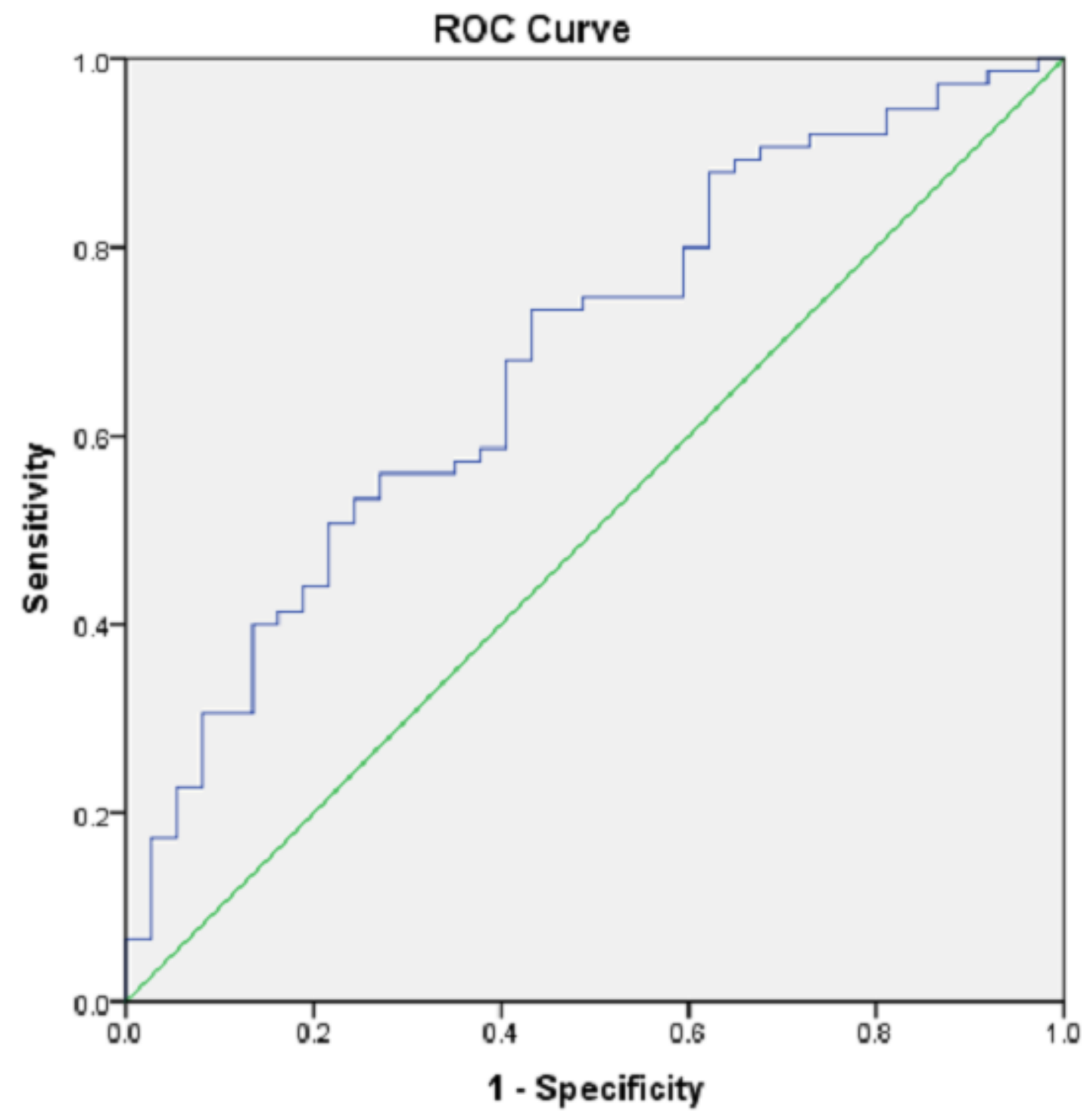

Figure 2

Receiver-operating characteristic curve of uterus volume against detection of live birth. 